\title{
Simplices with Equiareal Faces
}

\author{
P. McMullen \\ Department of Mathematics, University College London, \\ Gower Street, London WC1E 6BT, England \\ p.mcmullen@ucl.ac.uk
}

\begin{abstract}
If all the edges of a $d$-simplex $T$ have the same length, then $T$ is regular. However, if $d \geq 3$, then it is clear that the facets of $T$ may have the same $(d-1)$-volume without $T$ being regular. Here, the question of the extent to which the equality of $r$-volumes of the $r$-faces of $T$ implies regularity of $T$ is investigated, the case $r=d-2$ proving most fruitful.
\end{abstract}

\section{Introduction}

In private communications, Horst Martini and Robert Connelly have both raised the following question. Let $1 \leq r \leq d-1$, and let $T$ be a $d$-simplex, all of whose $r$-faces have the same $r$-volume; we then say that $T$ is $r$-equiareal. It is natural to ask: is an $r$-equiareal $d$-simplex necessarily regular? In what follows, we give a partial solution of this problem, and pose some resulting further questions.

Of course, the case $r=1$ of the problem is trivial-if all the edges of a $d$-simplex $T$ have the same length, then $T$ is regular. Henceforth, therefore, we may assume that $r \geq 2$.

It is also fairly clear that, if $d \geq 3$, then there are many simplices whose facets all have the same area $((d-1)$-volume); we discuss this case in detail in Section 4 . The complete solution in case $d=3$ then implies that 2-equiareal $d$-simplices are regular if $d \geq 4$. The interest therefore centres on the cases $3 \leq r \leq d-2$ (and hence $d \geq 5$ ). The central question here is whether, for $d \geq 5$, a $(d-2)$-equiareal $d$-simplex is necessarily also $(d-1)$-equiareal. We should say at once that there is no obvious reason why this should be so. However, the only examples thrown up by computer searches (admittedly in small dimensions) all have this property. We discuss this question in Section 6.

Conversely, though, in Section 5 we show how to construct many $(d-2)$-equiareal $d$-simplices; naturally, in view of what we have just said, these are also $(d-1)$-equiareal. There are non-regular examples for $d=5$ and each $d \geq 7$, and even an example for 
$d=11$ which has no symmetry whatsoever. Further, as we show in Section 6, if the central question has a positive answer, then an $r$-equiareal $d$-simplex would be regular whenever $1 \leq r \leq d-3$.

General references to the theory of convex polytopes are [1] and [4]; results about polytopes for which no specific citation is made can be found there.

\section{Standardization}

We begin the discussion with some preliminary remarks, and a useful normalization convention.

A $d$-simplex in $\mathbb{E}^{d}$ may be written in the form

$$
T=\left\{x \in \mathbb{E}^{d} \mid\left\langle x, u_{i}\right\rangle \leq \eta_{i} \text { for } i=0, \ldots, d\right\},
$$

where $U=\left(u_{0}, \ldots, u_{d}\right)$ is a set of vectors which positively span $\mathbb{E}^{d}$. (It is convenient always to think of $U$ as ordered. Furthermore, we often also identify $U$ with the $(d+1) \times d$ matrix whose rows are $u_{0}, \ldots, u_{d}$, coordinatized with respect to an orthonormal basis of $\mathbb{E}^{d}$.) We call $\eta_{j}$ the support parameter of $T$ corresponding to $u_{j}$. Note that if we replace $u_{j}$ by some positive multiple $\lambda u_{j}$, then we must similarly replace $\eta_{j}$ by $\lambda \eta_{j}$.

Up to a constant factor, there is a unique linear relation among $u_{0}, \ldots, u_{d}$, say

$$
\sum_{i=0}^{d} \alpha_{i} u_{i}=o
$$

the zero vector. If the $u_{j}$ are unit vectors, then the Minkowski relation for areas and normal vectors of polytopes says that we may take $\alpha_{j}$ to be the area $((d-1)$-volume) $\operatorname{vol}_{d-1} S_{j}$ of the corresponding facet $S_{j}$ of $T$.

Here, we find it more convenient to take an alternative normalization. If we assume the vectors $u_{j}$ scaled so that

$$
\sum_{i=0}^{d} u_{i}=o,
$$

then we call the set $U$ standardized. This clearly means that, for some fixed $\kappa>0$,

$$
\left\|u_{j}\right\|=\kappa \operatorname{vol}_{d-1} S_{j}
$$

for $j=0, \ldots, d$. If we wish, we may suppose that $\kappa=1$, but later other scalings will prove more convenient.

All simplices with $U$ as their sets of normal vectors are homothetic. One advantage of standardizing $U$ is the following.

Proposition 2.1. Let $T$ be a d-simplex with $U$ as its standardized set of normal vectors. Then the expression of $T$ in (2.1) is such that $\eta_{j}=\eta$ is constant for $j=0, \ldots, d$ if and only if the centroid of $T$ is $o$. 
Proof. For $j=0, \ldots, d$, let the vertex of $T$ opposite $S_{j}$ be $a_{j}$, so that we have

$$
\left\langle u_{i}, a_{j}\right\rangle=\eta_{i}
$$

for each $i \neq j$. Thus

$$
\left\langle u_{j}, a_{j}\right\rangle=-\sum_{i \neq j} \eta_{i}
$$

for each $j=0, \ldots, d$. It follows that the condition for the centroid of $T$ to be $o$, namely $\sum_{i=0}^{d} a_{i}=o$, reduces to

$$
-\sum_{i \neq j} \eta_{i}=-d \eta_{j}
$$

for each $j=0, \ldots, d$. It is easy to see that the only solutions satisfy $\eta_{0}=\cdots=\eta_{d}$, as claimed.

We say that $T$ is in standardform if its normal vectors are standardized and its support parameters are equal (so that $o$ is the centroid of $T$ ). For the most part, the actual common value of the support parameters will not interest us, so that the standardized set $U$ of normal vectors determines $T$ up to positive scalar multiple.

In fact, we shall invariably be concerned with the congruence classes of simplices under euclidean isometry. In this case, the actual vectors $u_{i}$ can be replaced by the matrix $U U^{\top}=\left(\left\langle u_{i}, u_{j}\right\rangle\right)$ of their inner products. This is explained by

Proposition 2.2. There is a one to one correspondence between standardized sets of normal vectors to $d$-simplices and positive semi-definite symmetric $(d+1) \times(d+1)$ matrices of rank $d$ whose rows sum to the zero vector $o$.

Proof. Certainly the matrix $U U^{\top}$ has the given properties. For the converse, let $Z$ be such a matrix. Then there exists an invertible $(d+1) \times(d+1)$ matrix $V$ such that

$$
V Z V^{\top}=\left[\begin{array}{cc}
I & o^{\top} \\
o & 0
\end{array}\right],
$$

where $I$ is the $d \times d$ identity matrix. We see at once that the $(d+1) \times d$ matrix

$$
U:=V^{-1}\left[\begin{array}{l}
I \\
o
\end{array}\right]
$$

satisfies $Z=U U^{\top}$, as required.

An important criterion for $r$-equiareality of a simplex $T$ will be expressed in terms of the polar simplex $T^{*}$. Recall that if $o \in \operatorname{int} T$ (the interior of $T$ ), then its polar is

$$
T^{*}:=\left\{y \in \mathbb{E}^{d} \mid\langle x, y\rangle \leq 1 \text { for all } x \in T\right\} .
$$

Proposition 2.3. If the $d$-simplex $T$ is in standard form, then so is its polar $T^{*}$. 
Proof. This is clear, since if there is a constant $\eta>0$, such that $\eta_{j}=\eta$ for $j=0, \ldots, d$, then the set of normals to $T^{*}$ is just $U^{*}=\left(\eta^{-1} a_{0}, \ldots, \eta^{-1} a_{d}\right)$, and we know that $\sum_{i=0}^{d} a_{i}=o$ from the definition of standard form.

As we shall see in Section 3, equiareality for $T$ translates into a very similar property for $T^{*}$.

\section{Volumes of Faces}

Let $T$ be a $d$-simplex in standard form. Thus the normal vector $u_{j}$ to the $j$ th facet $S_{j}$ of $T$ is normalized so that (2.2) holds, and $T$ itself is then of the form

$$
T=T(U, \eta):=\left\{x \in \mathbb{E}^{d} \mid\left\langle x, u_{i}\right\rangle \leq \eta \text { for } i=0, \ldots, d\right\}
$$

for some $\eta>0$. We calculate the volumes of the various faces of $T$ in terms of $U=$ $\left(u_{0}, \ldots, u_{d}\right)$ and $\eta$. Such expressions were previously found in [2], but the earlier forms are not well suited to our applications. In any case, we give a quicker proof here.

If $\left\{a_{1}, \ldots, a_{r}\right\} \subseteq \mathbb{E}^{d}$ is any set, then we define

$$
\operatorname{Det}\left(a_{1}, \ldots, a_{r}\right):=\operatorname{det}\left(\left\langle a_{i}, a_{j}\right\rangle\right)^{1 / 2},
$$

the $r$-volume of the parallelotope

$$
\left\{\sum_{i=1}^{r} \lambda_{i} a_{i} \mid 0 \leq \lambda_{i} \leq 1 \text { for } i=1, \ldots, r\right\},
$$

as in the geometry of numbers. (Of course, this volume is positive if and only if the vectors $a_{1}, \ldots, a_{r}$ are linearly independent.) Let $\Delta:=\operatorname{Det}\left(u_{1}, \ldots, u_{d}\right)$. Then we observe the following.

Lemma 3.1. If $j(1), \ldots, j(d)$ are any distinct indices, then

$$
\operatorname{Det}\left(u_{j(1)}, \ldots, u_{j(d)}\right)=\Delta .
$$

Proof. Indeed, let $j(0), \ldots, j(d)$ be $0, \ldots, d$ in some order. Then

$$
\begin{aligned}
\operatorname{Det}\left(u_{j(0)}, u_{j(2)}, \ldots, u_{j(d)}\right) & =\operatorname{Det}\left(-\sum_{i=1}^{d} u_{j(i)}, u_{j(2)}, \ldots, u_{j(d)}\right) \\
& =\operatorname{Det}\left(-u_{j(1)}, u_{j(2)}, \ldots, u_{j(d)}\right) \\
& =\operatorname{Det}\left(u_{j(1)}, u_{j(2)}, \ldots, u_{j(d)}\right),
\end{aligned}
$$

and the lemma follows at once.

Observe, by the way, that such calculations as occur in the proof are easily justified from the original definition; we employ them again below. An alternative way of looking 
at this result is the following. Since the only eigenvectors of the $(d+1) \times(d+1)$ matrix $U U^{\top}$ corresponding to the eigenvalue 0 are multiples of $e:=(1,1, \ldots, 1)$, the adjoint of $U U^{\top}$ must be $\Delta J$, with $J$ the $(d+1) \times(d+1)$ matrix all of whose entries are 1 . Of course, $\operatorname{Det}\left(u_{0}, u_{1}, \ldots, u_{d}\right)=0$.

In preparation for our general result, we first calculate the volume of $T$ in terms of the normal vectors $u_{j}$ and the support parameter $\eta$.

Lemma 3.2. The volume of the standard simplex $T$ is

$$
\operatorname{vol}_{d} T=\frac{(d+1)^{d} \eta^{d}}{d ! \operatorname{Det}\left(u_{1}, \ldots, u_{d}\right)} .
$$

Proof. We adopt our earlier notation, so that $a_{j}$ is the vertex of $T$ opposite the facet $S_{j}$ with outer normal $u_{j}$. We see easily that, for $i, j=1, \ldots, d$,

$$
\left\langle a_{i}-a_{0}, u_{j}\right\rangle=-(d+1) \eta \delta_{i j}
$$

with $\delta_{i j}$ the usual Kronecker delta. There follows immediately

$$
\operatorname{Det}\left(a_{1}-a_{0}, \ldots, a_{d}-a_{0}\right) \operatorname{Det}\left(u_{1}, \ldots, u_{d}\right)=((d+1) \eta)^{d} .
$$

Since $\operatorname{vol}_{d} T=\operatorname{Det}\left(a_{1}-a_{0}, \ldots, a_{d}-a_{0}\right) / d$ !, we obtain the result we sought.

Next we have the easily proved

Lemma 3.3. Suppose that the orthogonal projection of $u_{j(i)}$ on the orthogonal complement of $\operatorname{lin}\left\{u_{j(r+1)}, \ldots, u_{j(d)}\right\}$ is $v_{j(i)}$. Then, for any distinct indices $j(1), \ldots, j(d)$,

$$
\operatorname{Det}\left(u_{j(1)}, \ldots, u_{j(d)}\right)=\operatorname{Det}\left(v_{j(1)}, \ldots, v_{j(r)}\right) \operatorname{Det}\left(u_{j(r+1)}, \ldots, u_{j(d)}\right) .
$$

We now have the main result.

Theorem 3.4. For distinct indices $j(r+1), \ldots, j(d)$, the $r$-volume of the $r$-face $F:=$ $S_{j(r+1)} \cap \cdots \cap S_{j(d)}$ is given by

$$
\operatorname{vol}_{r} F=\frac{(d+1)^{r} \eta^{r} \operatorname{Det}\left(u_{j(r+1)}, \ldots, u_{j(d)}\right)}{r ! \operatorname{Det}\left(u_{1}, \ldots, u_{d}\right)} .
$$

Proof. Let $j(0), \ldots, j(r)$ be the missing indices. Then $F=\operatorname{conv}\left(a_{j(0)}, \ldots, a_{j(r)}\right)$, with the usual notation, so that $\operatorname{vol}_{r} F=\operatorname{Det}\left(a_{j(1)}-a_{j(0)}, \ldots, a_{j(r)}-a_{j(0)}\right) / r$ !. With $v_{j(i)}$ defined as in Lemma 3.3, we have

$$
\left\langle a_{j(i)}-a_{j(0)}, v_{j(k)}\right\rangle=-(d+1) \eta \delta_{i k}
$$

for $i, k=1, \ldots, r$, since $\left\langle a_{j(i)}-a_{j(0)}, v_{j(k)}\right\rangle=0$ for $i=1, \ldots, r$ and $k=r+1, \ldots, d$. Exactly similar calculations to those of Lemma 3.2 then lead to

$$
\operatorname{vol}_{r} F=\frac{(d+1)^{r} \eta^{r}}{r ! \operatorname{Det}\left(v_{j(1)}, \ldots, v_{j(r)}\right)} .
$$

Substituting for $\operatorname{Det}\left(v_{j(1)}, \ldots, v_{j(r)}\right)$ from Lemma 3.3 yields the theorem at once. 
If a $d$-simplex $T$ is in standard form, then we may take its set $U$ of normal vectors as the vertex-set of the polar simplex $T^{*}$, up to some positive scaling factor. In fact,

$$
T(U, \eta)^{*}=\eta^{-1} \operatorname{conv}\left\{u_{0}, \ldots, u_{d}\right\} .
$$

To an $r$-face $F$ of $T$ then corresponds a $(d-r-1)$-face $\widehat{F}$ of $T^{*}$, and hence a $(d-r)$ simplex

$$
\bar{F}:=\operatorname{conv}(\widehat{F} \cup\{o\}) .
$$

The equiareality criterion is then

Theorem 3.5. The $d$-simplex $T$ is $r$-equiareal if and only if all the $(d-r)$-simplices $\bar{F}$ corresponding to the $r$-faces of $T$ have the same $(d-r)$-volume.

Proof. This is clear when we observe that, if $F=S_{j(r+1)} \cap \cdots \cap S_{j(d)}$ as before, then $\bar{F}=\eta^{-1} \operatorname{conv}\left\{o, u_{j(r+1)}, \ldots, u_{j(d)}\right\}$, so that

$$
\begin{aligned}
\operatorname{vol}_{d-r} \bar{F} & =\frac{\operatorname{Det}\left(\eta^{-1} u_{j(r+1)}, \ldots, \eta^{-1} u_{j(d)}\right)}{(d-r) !} \\
& =\frac{\operatorname{Det}\left(u_{j(r+1)}, \ldots, u_{j(d)}\right)}{(d-r) ! \eta^{d-r}}
\end{aligned}
$$

In other words, from Theorem 3.4,

$$
\begin{aligned}
\operatorname{vol}_{r} F & =\frac{(d+1)^{r} \eta^{r} \operatorname{Det}\left(u_{j(r+1)}, \ldots, u_{j(d)}\right)}{r ! \operatorname{Det}\left(u_{1}, \ldots, u_{d}\right)} \\
& =\frac{(d+1)^{r}(d-r) ! \eta^{d}}{r ! \operatorname{Det}\left(u_{1}, \ldots, u_{d}\right)} \operatorname{vol}_{d-r} \bar{F}
\end{aligned}
$$

from which the criterion follows immediately.

Theorem 3.4 shows that $r$-equiareality imposes $\left(\begin{array}{l}d+1 \\ r+1\end{array}\right)$ conditions on the $u_{j}$ or, rather, in view of Proposition 2.2, on the matrix $Z:=U U^{\top}$. Thus it is far from surprising that, as we shall see in Section $4,(d-1)$-equiareality is frequent. However, with $(d-2)$-equiareality, we have exactly the same number of equations as variables, namely $\left(\begin{array}{l}d+1 \\ d-1\end{array}\right)+d+1=\left(\begin{array}{c}d+2 \\ 2\end{array}\right)$ on the $\left(\begin{array}{c}d+2 \\ 2\end{array}\right)$ distinct entries of $Z$; we should therefore expect finitely many solutions. (The same applies to the case $r=1$, but we recall that this implies regularity of $T$.) On the other hand, when $2 \leq r \leq d-3$, we have more equations than variables, and so we would be surprised to find any but trivial solutions (that is, again when $T$ is regular). We have already confirmed this for the case $r=2$. We investigate these problems in subsequent sections.

\section{Equiareal Facets}

In this section we use the term "equiareal" (without any qualification) to mean " $(d-1)$ equiareal". We shall see that equiareal simplices (in this sense) are very common. We begin with a general result. 
Theorem 4.1. The following conditions on a $d$-simplex $T$ are equivalent:

(a) $T$ is equiareal;

(b) the in-centre and centroid of $T$ coincide;

(c) the altitudes of $T$ are equal.

Proof. To see this, we take $T$ in standard form. For the equivalence of conditions (a) and (b), note that equality of facet areas just says that the standardized normal vectors have the same length, and since the support parameters are equal, this says that the facets of $T$ touch a sphere whose centre is the centroid of $T$. The equivalence of (a) (or (b)) with (c) is trivial.

As we said in Section 1 , if $d \geq 3$, then there are many different similarity classes of equiareal $d$-simplices. To see this, we make a simple observation. There is no harm in scaling such a simplex, so that its normal-set $U$ comprises unit vectors $u_{i}$, which satisfy (2.2). Of course, since $U$ is the normal-set to some simplex, the vectors $u_{i}$ must span $\mathbb{E}^{d}$ (linearly), and hence must be in linearly general position, meaning that no $d$ of them lie in any hyperplane through $o$.

Since $d \geq 3$, there are many ways of partitioning $\{0, \ldots, d\}$ into two sets $I$ and $J$, each of which contains at least two elements. Then

$$
\sum_{i \in I} u_{i}=-\sum_{i \in J} u_{i}=: w
$$

say, a non-zero vector. Let $\Phi$ be any sufficiently small rotation about $o$ which fixes the line through $w$, and define

$$
v_{i}:= \begin{cases}u_{i}, & \text { if } \quad i \in I, \\ u_{i} \Phi, & \text { if } \quad i \in J .\end{cases}
$$

Since $w \Phi=w$, it is clear that $V:=\left(v_{0}, \ldots, v_{d}\right)$ also consists of unit vectors satisfying (2.2), and since $\Phi$ is small, they remain in linearly general position (indeed, the smallness of $\Phi$ is irrelevant, as long as this condition holds). Observe that this idea actually gives continuous families of solutions.

We now look at some small values of $d$. We begin with $d=3$; here we have a complete characterization.

Theorem 4.2. A tetrahedron is equiareal if and only if its opposite pairs of edges have the same lengths.

Proof. First, let the tetrahedron $T$ be equiareal, with $U=\left(u_{0}, \ldots, u_{3}\right)$ its standardized set of normal vectors. Thus the vectors $u_{j}$ have the same length. Now we have (for example) $u_{0}+u_{1}=-\left(u_{2}+u_{3}\right)$, from which it is easy to see that the half-turn about the line joining $\pm\left(u_{0}+u_{1}\right)$ permutes the pairs $\left\{u_{0}, u_{1}\right\}$ and $\left\{u_{2}, u_{3}\right\}$. It follows that the opposite pairs of edges determined by the remaining four pairs of normals have the same length. The condition of the theorem follows at once.

Conversely, if the opposite edges of $T$ have the same length, then its four faces are congruent, and so have the same area. This completes the proof. 
Of course, tetrahedra with all facets having a fixed area can have volumes varying from 0 to the maximum, at a regular tetrahedron. This is in contrast with the result of Sabitov [3], which says that a flexible polyhedron, which can vary continuously while keeping its edge-lengths fixed, has a fixed volume.

There is an immediate consequence of Theorem 4.2. If $T$ is a 2-equiareal 4-simplex, with vertices $a_{0}, \ldots, a_{4}$, say, then consideration of the facets of $T$ which contain a given edge conv $\left\{a_{i}, a_{j}\right\}$ shows that the opposite triangular face of $T$ is equilateral. Hence $T$ must be regular. An easy induction argument then leads to

Theorem 4.3. If $d \geq 4$, then a 2-equiareal $d$-simplex is regular.

We return to equiareality (the case $r=d-1$ ) for general $d \geq 4$. To focus our ideas, we pose the following question: given a $(d-1)$-simplex $S$, under what conditions is $S$ a facet of an equiareal $d$-simplex $T$, and to what extent is $T$ unique? When $d=3$, the question has a straightforward answer-the triangle $S$ must be acute-angled, and uniqueness is guaranteed by Theorem 4.2. We look at the general case first, and then describe specific examples when $d=4$.

Let $S_{0}:=S$ be a $(d-1)$-simplex with $\operatorname{vol}_{d-1} S=1$, situated with its centroid at $o$ in the hyperplane $H$ orthogonal to the unit vector $u_{0}$. Let $S$ have facets $R_{0 j}$ and corresponding standardized normals $v_{0 j}$, for $j=1, \ldots, d$. We try to make the $R_{0 j}$ ridges of an equiareal $d$-simplex $T$ lying in the half-space $H^{-}:=\left\{x \in \mathbb{E}^{d} \mid\left\langle x, u_{0}\right\rangle \leq 0\right\}$. So, we wish to find unit vectors $u_{1}, \ldots, u_{d}$ of the form

$$
u_{j}=\rho_{j} v_{0 j}-\sigma_{j} u_{0},
$$

which satisfy $\sum_{i=0}^{d} u_{i}=o$.

The first observation is that $\rho_{1}=\cdots=\rho_{d}=: \rho$, say, a constant, because $\sum_{i=1}^{d} v_{0 i}=$ $o$ is essentially the only linear relation among the $v_{0 j}$. Further, we must have $\sum_{i=1}^{d} \sigma_{i}=1$. Finally, the condition that $\left\|u_{j}\right\|=1$ is $\rho^{2}\left\|v_{j}\right\|^{2}+\sigma_{j}^{2}=1$ for $j=1, \ldots, d$.

Solving the last equations gives

$$
\sigma_{j}=\varepsilon_{j} \sqrt{1-\rho^{2}\left\|v_{0 j}\right\|^{2}}
$$

with $\varepsilon_{j}= \pm 1$ for $j=1, \ldots, d$. The range of possible $\rho$ is clearly

$$
0<\rho \leq \min \left\{\left\|v_{0 j}\right\|^{-1} \mid j=1, \ldots, d\right\}
$$

for a predetermined choice of the $\varepsilon_{j}$, there may or may not be a suitable $\rho$, namely one satisfying

$$
\sum_{i=1}^{d} \varepsilon_{i} \sqrt{1-\rho^{2}\left\|v_{0 j}\right\|^{2}}=1 .
$$

However, in principle, we may determine all the (finitely many) solutions of the equations. In fact, if we replace $S$ by its scaled copy

$$
\left\{x \in H \mid\left\langle x, v_{0 j}\right\rangle \leq 1 \text { for } j=1, \ldots, d\right\},
$$


and let the vertices of $T$ be $a_{0}, \ldots, a_{d}$ (with $a_{j}$ opposite $S_{j}$ as usual), then, for each $j \neq 0$, we have $\left\langle a_{0}, u_{j}\right\rangle=\left\langle a_{i}, u_{j}\right\rangle=\left\langle a_{i}, \rho v_{0 j}-\sigma_{j} u_{0}\right\rangle=\rho$, with any $i \neq 0, j$. Since $u_{0}=-\sum_{j=1}^{d} u_{j}$, there follows

$$
-\left\langle a_{0}, u_{0}\right\rangle=d \rho
$$

Because $u_{0}$ is a unit vector, this says that $T$ has altitude $d \rho$.

For our first example, we choose as $S$ a tetrahedron in the linear hyperplane $H$ of $\mathbb{E}^{4}$ orthogonal to $e_{4}$; we take $u_{0}:=-e_{4}$. In $H$, the normal-set to $S$ is given by

$$
v_{0 j}:= \begin{cases}-e_{j}, & \text { if } j=1,2,3 \\ e_{1}+e_{2}+e_{3}, & \text { if } j=4\end{cases}
$$

A brief inspection shows that we only obtain a solution for $\rho$ if exactly one of the $\varepsilon_{j}$ is -1 for $j=1,2,3$ (and $\varepsilon_{4}=1$ ). This yields three equiareal 4-simplices $T$ of which $S$ is a facet.

We now vary $S$ a little. We redefine

$$
v_{0 j}:= \begin{cases}-\alpha_{j} e_{j}, & \text { if } j=1,2,3 \\ \alpha_{1} e_{1}+\alpha_{2} e_{2}+\alpha_{3} e_{3}, & \text { if } j=4,\end{cases}
$$

with $\alpha_{1}>\alpha_{2}>\alpha_{3}>0$ all close enough to 1 . The same analysis applies, and we obtain three mutually non-congruent equiareal 4-simplices of which $S$ is a facet. Indeed, the three corresponding values of $\rho$ are distinct, and so, by the remark above, the resulting 4-simplices have different volumes.

Similar examples may be constructed for each $d \geq 5$, with the conclusion that, even if an equiareal $d$-simplex exists with a given $(d-1)$-simplex as facet, generally it will not be unique.

\section{Equiareal Ridges}

We now show how to construct $(d-2)$-equiareal $d$-simplices. The examples presented here demonstrate that such simplices need not have much (or indeed any) symmetry. We begin with

Lemma 5.1. Let $T$ be a $(d-1)$-equiareal $d$-simplex. Then $T$ is $(d-2)$-equiareal if and only if there is some angle $\vartheta$, such that each dihedral angle of $T$ is either $\vartheta$ or $\pi-\vartheta$.

Proof. Let $T$ have standardized normal vectors $u_{0}, \ldots, u_{d}$. Since $T$ is $(d-1)$-equiareal, we may assume that $\left\|u_{i}\right\|=1$ for each $i=0, \ldots, d$. Let the dihedral angle between the facets $S_{i}$ and $S_{j}$ of $T$ be $\vartheta_{i j}$. The criterion of Theorem 3.5 for $(d-2)$-equiareality says that, for $i \neq j$,

$$
\sin ^{2} \vartheta_{i j}=1-\cos ^{2} \vartheta_{i j}=\left\|u_{i}\right\|^{2}\left\|u_{j}\right\|^{2}-\left\langle u_{i}, u_{j}\right\rangle^{2}=\operatorname{Det}\left(u_{i}, u_{j}\right)^{2}
$$

is constant, independent of $i$ and $j$. In other words, for some fixed angle $\vartheta$, we have $\vartheta_{i j}=\vartheta$ or $\pi-\vartheta$. The converse is clear, which proves the lemma. 
Henceforth, we suppose that the $d$-simplex $T$ is $(d-1)$ - and $(d-2)$-equiareal, and also that the angle $\vartheta$ of Lemma 5.1 is acute (it clearly cannot be $\pi / 2$ ). Take any facet $S_{i}$ of $T$. Let $m$ of the dihedral angles $\vartheta_{i j}$ at $S_{i}$ be obtuse, and the remaining $d-m$ acute. Let each $S_{j}$ have area $v$. Consider the areas of the projections of the $S_{j}$ with $j \neq i$ on the hyperplane aff $S_{i}$; these are counted with sign \pm 1 according as $\vartheta_{i j}$ is acute or obtuse. Hence we have

$$
v=\sum_{j \neq i} v \cos \vartheta_{i j}=(d-m) v \cos \vartheta-m v \cos \vartheta=(d-2 m) v \cos \vartheta
$$

giving

$$
\cos \vartheta=\frac{1}{d-2 m}
$$

Observe that $m$ must thus be the same for each facet $S_{i}$ of $T$, and that, to avoid degeneracy, we must have $d-2 m>1$, and hence $d-2 m \geq 2$.

We deduce the following angle criterion:

Theorem 5.2. Let $T$ be $a(d-1)$ - and $(d-2)$-equiareal $d$-simplex. Then there is some number $m \geq 1$ with $d \geq 2 m+2$, such that at each facet of $T$ there are $d-m$ dihedral angles $\vartheta$ and $m$ dihedral angles $\pi-\vartheta$, where

$$
\cos \vartheta=\frac{1}{d-2 m}
$$

We now introduce the obtuseness graph $G$ of $T$; its nodes are the facets of $T$, and there is a branch joining two nodes if the dihedral angle between the corresponding facets is obtuse. Thus $G$ is an $m$-regular graph (that is, each node of $G$ has degree $m$ ), with $m$ given by Theorem 5.2. If $M:=M(G)$ is the adjacency matrix of $G$ (so that $M$ is the $(d+1) \times(d+1)$ matrix, whose $(i, j)$ entry is 1 or 0 according as the nodes $i$ and $j$ are or are not joined by a branch), then the matrix of inner products between the pairs of normal vectors is given by

$$
(d-2 m) U U^{\top}=(d-2 m+1) I-J+2 M .
$$

Here, $I$ is the $(d+1) \times(d+1)$ identity matrix, and $J$ is the $(d+1) \times(d+1)$ matrix all of whose entries are 1.

We now have the adjacency matrix criterion:

Theorem 5.3. There exist unit vectors $u_{0}, \ldots, u_{d}$ (necessarily in linearly general position) which satisfy (5.2) if and only if the matrix $K:=(d-2 m+1) I-J+2 M$ is positive semi-definite of rank $d$.

Proof. This is clear; the sole eigenvector with eigenvalue 0 is $e=(1, \ldots, 1)$.

Before we come to specific examples, we discuss this situation in general terms. Let $M$ be the adjacency matrix of an $m$-regular graph $G$ with $d+1$ nodes. Thus $M$ is a symmetric $(0,1)$-matrix, with constant row (and column) sum $m$. Now clearly $M$ has 
the eigenvector $e=(1, \ldots, 1)$, with eigenvalue $m$; we call this eigenvector trivial. Let $v=\left(\beta_{1}, \ldots, \beta_{a}\right)$ be any eigenvector of $M$. If $\beta:=\max \left\{\left|\beta_{i}\right| \mid i=1, \ldots, d+1\right\}$, with the maximum achieved at $i=j$, say, then, by considering the $j$-coordinate of $b M$, we conclude that the corresponding eigenvalue $\mu$ satisfies

$$
|\mu \beta| \leq m|\beta|
$$

giving $|\mu| \leq m$.

We ask when $M$ gives rise to a $(d-2)$-equiareal $d$-simplex. We employ the adjacency matrix criterion of Theorem 5.3. The trivial eigenvector of $M$ is also one of $K$, with eigenvalue 0 . Thus we need only look at a non-trivial eigenvector $v$ of $M$, belonging to the eigenvalue $\mu$ say, for which $v J=o$ (we may always suppose that $v$ is orthogonal to $e$ ). The corresponding eigenvalue $\lambda$ of $K$ is given by

$$
\lambda v=v K=(d-2 m+1) v-v J+2 v M=(d-2 m+1+2 \mu) v,
$$

or $\lambda=d-2 m+1+2 \mu$. In view of $|\mu| \leq m$, we see that we can ensure that $\lambda>0$, if $d>2 m-1+2 \max |\mu|$, or $d \geq 4 m$.

In fact, we can improve on this a little. The adjacency matrix $M$ can have eigenvalue $\mu=-m$ if and only if $G$ has a bipartite component. (This can be seen by a similar argument to that giving $\max |\mu|$; we thank Imre Leader for bringing this fact to our attention.) In other words, if $G$ has no bipartite component, then, whenever $d \geq 4 m-1$, we have

$$
\lambda=d-2 m+1+2 \mu \geq 4 m-1-2 m+1+2 \mu=2(m+\mu)>0,
$$

Summarizing, we have

Theorem 5.4. Let $G$ be an $m$-regular graph on $d+1$ nodes. Then $G$ is the obtuseness graph of a $(d-1)$ - and $(d-2)$-equiareal $d$-simplex whenever $d \geq 4 m$, or, if $G$ has no bipartite component, whenever $d \geq 4 m-1$.

In particular cases, as we shall see below, an even smaller (relative) value of $d$ will often work.

We now apply these techniques. First, we give a direct construction (this provided us with our initial examples). Pick any proper divisor $m+1$ of $d+1$, such that $m \leq \frac{1}{2}(d-2)$ (thus the quotient 2 is not permitted; this also implies that $d \geq 5$ ). Write $n+1=$ $(d+1) /(m+1)$, and let $L, M_{0}, \ldots, M_{n}$ be mutually orthogonal linear subspaces of $\mathbb{E}^{d}$ of dimensions $n, m, \ldots, m$, respectively. (Note that $n+(n+1) m=d$, as required.) In $L$, let $v_{0}, \ldots, v_{n}$ be the unit normals to some regular $n$-simplex, and similarly in each $M_{i}$ let $w_{i 0}, \ldots, w_{i m}$ be the unit normals to some regular $m$-simplex. Finally, for each $i=0, \ldots, n$ and $j=0, \ldots, m$, and $\lambda, \mu>0$ to be determined, define

$$
u_{i j}:=\lambda v_{i}+\mu w_{i j}
$$

We first scale so that each $u_{i j}$ is a unit vector, which implies that

$$
\lambda^{2}+\mu^{2}=1 .
$$


Let $\vartheta_{i j}$ be the dihedral angle of the resulting simplex $T$ at its ridge $((d-2)$-face) $R_{i j}=S_{i} \cap S_{j}$, so that we shall have to have $\vartheta_{i j}=\vartheta$ or $\pi-\vartheta$ for some $\vartheta$ with $0<\vartheta<\pi / 2$. We can ensure this by setting

$$
\lambda^{2}-\frac{\mu^{2}}{m}=\left\langle u_{i j}, u_{i k}\right\rangle=\cos \vartheta
$$

for $i=0, \ldots, m$ and $0 \leq j<k \leq n$, and

$$
-\frac{\lambda^{2}}{n}=\left\langle u_{h j}, u_{i k}\right\rangle=-\cos \vartheta
$$

for $0 \leq h<i \leq m$, and any $j$ and $k$. It is easy to see that these equations are satisfied by

$$
\lambda^{2}=\frac{n}{d-2 m}, \quad \mu^{2}=\frac{d-2 m-n}{d-2 m}, \quad \cos \vartheta=\frac{1}{d-2 m}
$$

(The last equation is just as expected.) In other words, we have a $(d-2)$-equiareal $d$ simplex, whenever $d+1=(m+1)(n+1)$ for some $m$ and $n$ satisfying $1 \leq m \leq \frac{1}{2}(d-2)$ and $n \geq 2$.

In this example, the obtuseness graph $G$ has $n+1$ components, each of which is a complete graph on $m+1$ nodes. Notice that the case $n=2$, with $d=3 m+2$, is not covered by Theorem 5.4 .

Next, we fill most of the gaps in the range of values of $d$ covered by this construction. Let the obtuseness graph $G$ consist of a single circuit of $d+1$ nodes and edges in order $0,1, \ldots, d, 0$; thus $m=2$. Then $(d-3) I+J-2 M$ is a circulant matrix, whose nonzero eigenvalues are $d-3+4 \cos (2 k \pi /(d+1))$ (corresponding to the eigenvectors $\left(1, \omega^{k}, \omega^{2 k}, \ldots, \omega^{d k}\right)$ with $\left.\omega:=e^{2 i \pi /(d+1)}\right)$ for $k=1, \ldots, d$. For $d \geq 8$, these are always positive, as required. The case $d=7$, for which $k=4$ is not allowed, has been dealt with by the case $m=1$ of the previous construction.

However, for $d=6$, we have

$$
\cos (6 \pi / 7)<-\frac{3}{4}
$$

giving a negative eigenvalue. It is not too hard to see that there is no obtuseness graph on seven nodes which can make our construction work. Only $m=2$ is permitted (because we need $2 m \leq 7-2=5$ ), and the two possibilities for $G$ are a single heptagon (the case just considered), and a triangle and a square (the latter component being bipartite, which brings Theorem 5.4 into play).

Theorem 5.4 also permits an alternative obtuseness graph when $d=7$, namely that with two circuits, a triangle and a pentagon. Two squares, on the other hand, are excluded.

Finally, as another example of what Theorem 5.4 will do for us, we take $m=3$. It is not too hard to find a connected 3-regular graph on 12 nodes (even planar) which is not bipartite, and has no non-trivial automorphisms. (We need $d$ odd, because $m$ is odd.) 
For example, we can take the graph to be

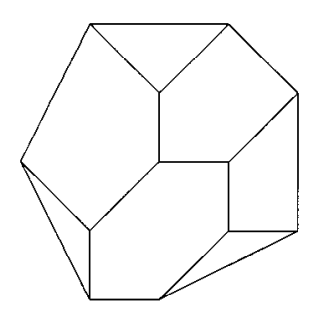

Since $11 \geq 4 \cdot 3-1$, and the graph has odd circuits (and so cannot be bipartite), Theorem 5.4 tells us that the corresponding 11-simplex is 9-equiareal. However, as is easy to see, the simplex has no non-trivial symmetries since the graph has none.

\section{Open Questions}

The constructions of Section 5, which give $(d-2)$-equiareal $d$-simplices which are not regular, lead us to pose several questions. The examples are all of one specific kind, and it is unclear whether such simplices exist which are not of this kind. The constructions thus raise what we call the central question.

Question 6.1. Let $d \geq 5$. Is a $(d-2)$-equiareal $d$-simplex necessarily also $(d-1)$ equiareal?

There is no obvious reason why the central question should have a positive answer (which is why we have posed it as a question rather than a conjecture). As we remarked in Section 1, a computer enumeration (by Robert Connelly) in small dimensions only produced $(d-1)$-equiareal examples. A $(d-2)$-equiareal $d$-simplex which is not $(d-1)$ equiareal would have to satisfy a number of somewhat curious extra conditions, but we have not (so far) found any inconsistency in them. Indeed, as we saw in Section 5, as $d$ gets larger, a $(d-2)$-equiareal $d$-simplex may be less and less regular, until at $d=11$ it need have no symmetry whatsoever. Thus it is conceivable that, for even larger $d$, the property of $(d-1)$-equiareality may be lost as well.

Now, once again, let $T$ be a $(d-1)$ - and $(d-2)$-equiareal $d$-simplex. Let $G$ be the obtuseness graph of $T$, with degree $m$ at each node. Since $m \leq \frac{1}{2}(d-2)$, given any two nodes $i$ and $j$, say, there is a third node $k$ which is joined in $G$ to neither $i$ nor $j$. The normal vectors to the facets of $T$ may be chosen to be unit vectors, and (as we saw in Section 5) the corresponding normal vector $v_{i j}$ to the facet (ridge) $R_{i j}=S_{i} \cap S_{j}$ of $S_{i}$ at $S_{j}$ is then a vector of length $\sin \vartheta$ (with $\cos \vartheta=1 /(d-2 m)$ ). Hence the dihedral angle $\varphi_{i j k}=\varphi_{i k j}$ in the facet $S_{i}$ between its two facets $R_{i j}$ and $R_{i k}$ is given by

$$
-\cos \varphi_{i j k}=\frac{\left\langle v_{i j}, v_{i k}\right\rangle}{\sin ^{2} \vartheta}
$$




$$
\begin{aligned}
& =\frac{\left\langle u_{j}-\left\langle u_{i}, u_{j}\right\rangle u_{i}, u_{k}-\left\langle u_{i}, u_{k}\right\rangle u_{i}\right\rangle}{\sin ^{2} \vartheta} \\
& =\frac{\left\langle u_{j}, u_{k}\right\rangle-\left\langle u_{i}, u_{j}\right\rangle\left\langle u_{i}, u_{k}\right\rangle}{1-\cos ^{2} \vartheta} .
\end{aligned}
$$

Now $\left\langle u_{i}, u_{k}\right\rangle=\left\langle u_{j}, u_{k}\right\rangle=-\cos \vartheta$, whereas $\left\langle u_{i}, u_{j}\right\rangle= \pm \cos \vartheta$ as the dihedral angle between $S_{i}$ and $S_{j}$ is obtuse or acute. The corresponding values are thus given by

$$
\cos \varphi_{i j k}=\left\{\begin{array}{rlrl}
\frac{-\cos \vartheta+\cos ^{2} \vartheta}{1-\cos ^{2} \vartheta} & =-\frac{\cos \vartheta}{1+\cos \vartheta} & \\
& =-\frac{1}{(d-1)-2(m-1)}, & & \text { if obtuse, } \\
\frac{-\cos \vartheta-\cos ^{2} \vartheta}{1-\cos ^{2} \vartheta} & =-\frac{\cos \vartheta}{1-\cos \vartheta} & \\
& =-\frac{1}{(d-1)-2 m}, & & \text { if acute. }
\end{array}\right.
$$

Observe that both possibilities must occur.

Closer inspection of what we have found above shows that the dihedral angles $\varphi$ of a facet $S$ of $T$ are given by

$$
\cos \varphi= \pm \frac{1}{d-1-2 m} \quad \text { or } \quad \pm \frac{1}{d-1-2(m-1)},
$$

with $m$ given by Theorem 5.2; observe that these angles then fix $m$. A particular choice of the dihedral angle of $T$ at any given facet of $S$ as $\vartheta$ or $\pi-\vartheta$ (with $\cos \vartheta=1 /(d-2 m)$ ) then determines all the other dihedral angles of $T$; only one of the two choices will give $m$ obtuse dihedral angles. In other words,

Theorem 6.2. If a $(d-1)$-simplex $S$ is a facet of a $(d-1)$ - and $(d-2)$-equiareal $d$-simplex $T$, then $T$ is unique up to congruence.

That is, we can effectively reconstruct $T$ from $S$.

It is thus natural to ask, more generally,

Question 6.3. Is there, up to congruence, at most one $(d-2)$-equiareal $d$-simplex, one of whose facets is a given $(d-2)$-equiareal $(d-1)$-simplex?

Of course, if the central Question 6.1 has a positive answer, then Theorem 6.2 shows that Question 6.3 has also.

We now glance at $r$-equiareal $d$-simplices, when $r<d-2$. However, since the central question is still open, the discussion is necessarily somewhat tentative. Nevertheless, it is worth posing the following

Conjecture 6.4. If $3 \leq r \leq d-3$, then an $r$-equiareal $d$-simplex is regular. 
We have already observed, in Section 3, that the normal vectors of an $r$-equiareal $d$-simplex, with $3 \leq r \leq d-3$, satisfy more more equations than they have degrees of freedom, and thus the conjecture is likely to hold.

It is clearly enough, to prove Conjecture 6.4, to consider the case $r=d-3$. (Clearly, if, for a given $r$, it holds for a particular dimension $d$, then it holds for all larger dimensions.) The results above establish

Theorem 6.5. If Question 6.3 (or Question 6.1) has a positive answer, then Conjecture 6.4 holds.

\section{References}

1. B. Grünbaum, Convex Polytopes. Wiley-Interscience, New York, 1967.

2. C. F. Klebaner, A. Sudbury and G. A. Watterson, The volume of simplices, or, find the penguin. J. Austral. Math. Soc. Ser. A 47 (1989), 263-268.

3. I. Kh. Sabitov, The volume as a metric invariant of polyhedra. Discrete Comput. Geom. 20 (1998), 405-425.

4. G. M. Ziegler, Lectures on Polytopes. Springer-Verlag, New York, 1994.

Received January 30, 1999. Online publication May 9, 2000.

Note added in proof. We have recently constructed examples of $(d-2)$-equiareal $d$ simplices which are not $(d-1)$-equiareal, even for rather small $d$, thus giving a negative answer to Question 6.1. Details will appear later. 